Die ökologische Steuerreform in Deutschland 
Stefan Bach - Christhart Bork - Michael Kohlhaas Christian Lutz - Bernd Meyer - Barbara Praetorius Heinz Welsch

\section{Die ökologische Steuerreform in Deutschland}

Eine modellgestützte Analyse ihrer Wirkungen auf Wirtschaft und Umwelt

Unter Mitarbeit von

Viola Ehrenheim und Katja Schumacher

Mit 17 Abbildungen und 88 Tabellen

Springer-Verlag Berlin Heidelberg $\mathrm{GmbH}$ 


\author{
Dr. Stefan Bach \\ Deutsches Institut \\ für Wirtschaftsforschung \\ Königin-Luise-Straße 5 \\ 14195 Berlin \\ sbach@diw.de \\ Dr. Christhart Bork \\ Adolfstraße 6 \\ 65185 Wiesbaden \\ c.bork@berlin.de \\ Michael Kohlhaas \\ Deutsches Institut \\ für Wirtschaftsforschung \\ Königin-Luise-Straße 5 \\ 14195 Berlin \\ mkohlhaas@diw.de \\ Dr. Christian Lutz \\ GWS mbH \\ Blumenthalstraße 41 \\ 49076 Osnabrück \\ lutz@gws-os.de
}

Professor Dr. Bernd Meyer

Universität Osnabrück

Fachbereich Wirtschaftswissenschaften

Rolandstraße 8

49076 Osnabrück

meyer@oec.uni-osnabrueck.de

Dr. Barbara Praetorius

Deutsches Institut

für Wirtschaftsforschung

Königin-Luise-Straße 5

14195 Berlin

bpraetorius@diw.de

Professor Dr. Heinz Welsch

Universität Oldenburg

Institut für Volkswirtschaftslehre I

26111 Oldenburg

welsch@uni-oldenburg.de

\section{ISBN 978-3-642-63307-2}

Die Deutsche Bibliothek - CIP-Einheitsaufnahme

Die ökologische Steuerreform in Deutschland: eine modellgestützte Analyse ihrer Wirkungen auf Wirtschaft und Umwelt / von Stefan Bach ... - Heidelberg: Physica-Verl., 2001

ISBN 978-3-642-63307-2 ISBN 978-3-642-57605-8 (eBook)

DOI $10.1007 / 978-3-642-57605-8$

Dieses Werk ist urheberrechtlich geschützt. Die dadurch begründeten Rechte, insbesondere die der Übersetzung, des Nachdrucks, des Vortrags, der Entnahme von Abbildungen und Tabellen, der Funksendung, der Mikroverfilmung oder der Vervielfältigung auf anderen Wegen und der Speicherung in Datenverarbeitungsanlagen, bleiben, auch bei nur auszugsweiser Verwertung, vorbehalten. Eine Vervielfältigung dieses Werkes oder von Teilen dieses Werkes ist auch im Einzelfall nur in den Grenzen der gesetzlichen Bestimmungen des Urheberrechtsgesetzes der Bundesrepublik Deutschland vom 9. September 1965 in der jeweils geltenden Fassung zulässig. Sie ist grundsätzlich vergütungspflichtig. Zuwiderhandlungen unterliegen den Strafbestimmungen des Urheberrechtsgesetzes.

(C) Springer-Verlag Berlin Heidelberg 2001

Ursprünglich erschienen bei Physica-Verlag Heidelberg 2001

Die Wiedergabe von Gebrauchsnamen, Handelsnamen, Warenbezeichnungen usw. in diesem Werk berechtigt auch ohne besondere Kennzeichnung nicht zu der Annahme, dass solche Namen im Sinne der Warenzeichen- und Markenschutz-Gesetzgebung als frei zu betrachten wären und daher von jedermann benutzt werden dürften. 


\section{Inhaltsverzeichnis}

Einleitung ................................................................................................. 1

2 Methoden und Grenzen der modellgestützten Analyse wirtschaftspolitischer Strategien........................................................ 3

2.1 Methoden und Grenzen ökonomischer Modellanalysen .................. 3

2.2 Gesamtwirtschaftliche Modelle .................................................... 5

2.3 Mikrosimulationsmodelle .......................................................... 7

3 Ökologische Steuerreform und wirtschaftliche Rahmenbedingungen................................... 9

3.1 Referenzszenario: Entwicklung der Rahmenbedingungen.............. 9

3.1.1 Vorbemerkungen ...................................................................... 9

3.1.2 Energiewirtschaftliche Rahmenbedingungen bei moderaten und bei höheren Rohölpreisen und Wechselkursen ............................ $\quad 10$

3.1.2.1 Rohölpreise ........................................................................ 10

3.1.2.2 Importpreise für Steinkohle und Erdgas..................................... 13

3.1.2.3 Entwicklung der Importpreise für Elektrizität............................... 14

3.1.2.4 Zusammenfassung der Annahmen für die künftige
Preisentwicklung

3.1.2.5 Entwicklung der Stromerzeugung im Inland sowie der Importe...... 17

3.2 Steuerszenario: Die ökologische Steuerreform in Deutschland ....... 21

3.2.1 Struktur und Steuersätze ........................................................... 21

3.2.2 Sonderregelungen und klimapolitisch flankierende Maßnahmen.... 26

3.2.3 Effektive Be- und Entlastungen durch die ökologische Steuerreform im Produzierenden Gewerbe und in der Landwirtschaft 
$4 \quad$ Simulationen: Modelle und Ergebnisse ............................................ 40

4.1 Das umweltökonometrische Modell PANTA RHEI III .................... 40

4.1.1 Kurzbeschreibung von PANTA RHEI III........................................ 40

4.1.2 Das Referenzszenario des Modells PANTA RHEI.......................... 45

4.1.3 Simulationsergebnisse mit dem Modell PANTA RHEI ................... 51

4.1.4 Alternatives Energiepreis- und Wechselkursszenario .................... 65

4.1.5 Simulationsergebnisse mit dem Modell PANTA RHEI für das Szenario höherer Energiepreise ....................................................... 69

4.2 Das umweltökonomische Simulationsmodell LEAN ......................... 77

4.2.1 Kurzbeschreibung des Modells LEAN ............................................. 77

4.2.2 Das Referenzszenario des Modells LEAN.......................................... 79

4.2.3 Simulationsergebnisse mit dem Modell LEAN ................................. 82

4.2.3.1 Methodik ................................................................................... $\quad 82$

4.2.3.2 Gesamteffekt der ökologischen Steuerreform .................................... 83

4.2.3.3 Dekompositionsanalyse der Effekte der ökologischen Steuerreform ........................................................................ 90

4.2.3.4 Alternatives Energiepreisszenario (Hochpreisszenario).................... 94

4.3 Verteilungswirkungen im Potsdamer Steuer- und TransferMikrosimulationsmodell.................................................................... 97

4.3.1 Überblick ................................................................................ 97

4.3.2 Das Modell.............................................................................. 98

4.3.3 Abbildung des Steuer- und Transfersystems ................................... 100

4.3.4 Verteilungswirkungen in den Steuerszenarien................................ 106

4.3.5 Zusammenfassung ................................................................ 123

5 Vergleich der Ergebnisse und Beurteilung der ökologischen Steuerreform........................................................................................ 124

5.1 Modelltheoretische Vorüberlegungen ............................................. 124

$5.2 \quad$ Gesamtwirtschaftliche Effekte ……………..................................... 126

$5.3 \quad$ Verteilungswirkungen ............................................................ 133

5.4 Energieverbrauch und Emissionen ...................................................... 137

$5.5 \quad$ Umweltpolitische Beurteilung ...................................................... 144

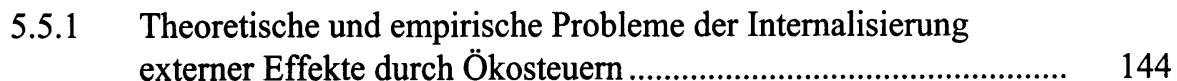

5.5.2 Sonderregelungen und komplementäre Instrumente.......................... 155

5.5.2.1 Zur Notwendigkeit eines Instrumentenmix ...................................... 155 
5.5.2.2 Substitution fossiler Energieträger durch erneuerbare Energien......

5.5.2.3 Effiziente Umwandlung durch Kraft-Wärme-Kopplung sowie Gas- und Dampfkraftwerke ........................................................... 162

5.6 Längerfristige Effekte der ökologischen Steuerreform .................... 169

5.6.1 Anreiz zu umweltentlastender Innovation .................................... 170

5.6.2 Induzierter Strukturwandel ....................................................... 173

$6 \quad$ Wirtschaftspolitische Schlussfolgerungen .......................................... 177

Verzeichnis der Abbildungen ................................................................................ 181

Verzeichnis der Tabellen......................................................................... 182

Literaturverzeichnis ............................................................................... 187 